\title{
INDIRECT EXCHANGE INTERACTIONS IN GRAPHENE
}

\author{
HYUNYONG LEE, ${ }^{1, *}$ EDUARDO R. MUCCIOLO,${ }^{2, \dagger}$ GEORGES BOUZERAR,${ }^{3,4, \ddagger}$ and \\ STEFAN KETTEMANN ${ }^{1,4, \S}$ \\ ${ }^{1}$ Division of Advanced Materials Science, Pohang University of Science and Technology \\ (POSTECH), Pohang 790-784, South Korea \\ ${ }^{2}$ Department of Physics, University of Central Florida, Orlando, Florida 32816, USA \\ ${ }^{3}$ Institut Néel, CNRS, départment MCBT, 25 avenue des Martyrs, BP 166, \\ 38042 Grenoble Cedex 09, France \\ ${ }^{4}$ School of Engineering and Science, Jacobs University Bremen, Bremen 28759, Germany \\ *hyunyongrhee@postechedu \\ ${ }^{\dagger}$ mucciolo@physics.ucf.edu \\ $\ddagger$ georges.bouzerar@grenoble.cnrs.fr \\ §s.kettemann@jacobs-university.de
}

\begin{abstract}
We study the Ruderman-Kittel-Katsuya-Yoshida (RKKY) interactions in graphene controlling the gate voltage and applying nonmagnetic disorder. It is found that oscillations of the RKKY interactions in undoped graphene are characterized by the interference of two neighbor Dirac nodes $\boldsymbol{K}$ and $\boldsymbol{K}^{\prime}$ in the first Brillouin zone and decays with $R^{-3}$ distance dependence. In the slightly doped graphene, a beating pattern, which consists of two characteristic wavevectors $\left(\boldsymbol{K}-\boldsymbol{K}\right.$ and $\left.\boldsymbol{k}_{\boldsymbol{F}}\right)$, starts to appear. The distance dependence in this regime shows a crossover from the $R^{-3}$ to $R^{-2}$. We present the effect of weak disorder on the RKKY interactions in diffusive regime. The arithmetic averaged interaction over disorder configurations decreases exponentially at distances exceeding the elastic mean free path, while the geometrical average(typical) value has the same power-law as the clean limit.
\end{abstract}

Keywords: RKKY; graphene.

PACS number: 71.70.Gm

\section{Introduction}

Many analytical and numerical studies on the RKKY interactions in clean graphene at Dirac (neutrality) point have been reported recently. ${ }^{1-4}$ Due to the particle-hole symmetry, in bipartite lattices including graphene, the RKKY interactions have always ferromagnetic correlation between the magnetic impurities on the same sublattice, but antiferromagnetic correlation between the ones on the different sublattice. ${ }^{1}$ Because of the pseudo gap at Dirac point, the distance $(R)$ dependence of the RKKY interactions in graphene is $1 / R^{3}$, which is unexpected behavior in 2 dimensional metal systems $\left(1 / R^{2}\right)$. Due to the presence of the two Dirac points $\boldsymbol{K}^{\left({ }^{\prime}\right)}=$ $( \pm 2 \pi / 3 \sqrt{3}, 2 \pi / 3)$ the RKKY interaction is governed by two different length scales, $\left|\boldsymbol{K}-\boldsymbol{K}^{\prime}\right|^{-1}$ and the Fermi wavelength $k_{F}^{-1}$, in doped (but near the Dirac point) 
(a)

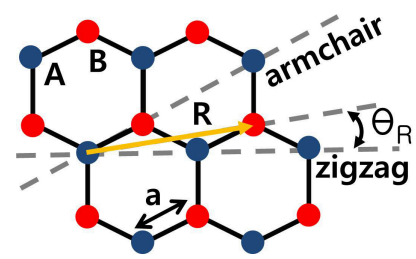

(b)

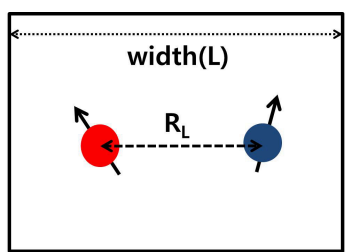

Fig. 1. (a) Schematic diagrams of graphene lattice. The two sublattices are denoted as A and B and the two representative directions (zigzag and armchair) are indicated as dashed gray lines. $\theta_{\boldsymbol{R}}$ is the angle between the displacement vector of the magnetic impurity $\boldsymbol{R}$ and the zigzag direction. (b) A lattice setup with the system size $L$ and the furthermost distance $R_{L}$ between impurity spins.

graphene. ${ }^{4}$ The on-site disorder scatters the phase of the electron's wave function and changes its amplitude randomly so that the period of the oscillation and its amplitude are altered comparing to the one in the clean system. In diffusive regime, consequently, the averaged RKKY interaction over disorder configurations decays exponentially on distances exceeding the mean free path scale $l_{e}$. However its geometrical average value has the same power law decaying as the clean sample ${ }^{8}$ so that weak disorder is not likely to cause any critical change in physical properties which derive from the RKKY interaction. We summarize the results obtained recently. ${ }^{8,9}$

\section{Model and method}

We start with a general expression for the RKKY exchange coupling constant in terms of the unperturbed (disorder-free) electronic Green's function $G^{(0)}\left(\mathbf{r}_{i}, \mathbf{r}_{j}, \omega\right){ }^{5}$

$$
\begin{aligned}
J_{\mathrm{RKKY}} & =J^{2} \frac{S(S+1)}{4 \pi S^{2}} \int d \omega f(\omega) \operatorname{Im}\left[G^{(0)}\left(\boldsymbol{r}_{j}, \boldsymbol{r}_{i}, \omega\right) \times G^{(0)}\left(\boldsymbol{r}_{i}, \boldsymbol{r}_{j}, \omega\right)\right] \\
& =J^{2} \frac{S(S+1)}{4 \pi S^{2}} \operatorname{Im} \int d \omega f(\omega) \times \sum_{n, m} \frac{F_{n m}^{i j}}{\left(E_{n}-\omega+i \delta\right)\left(E_{m}-\omega+i \delta\right)} .
\end{aligned}
$$

where $J$ is the local coupling constant between the localized magnetic impurities and the itinerant electrons, $S$ is the magnitude of the impurity spin, $i(j)$ is the site index of a magnetic impurity located at position $\boldsymbol{r}_{i}\left(\boldsymbol{r}_{j}\right), f(\omega)=\left[e^{(\omega-\mu) / T}+1\right]^{-1}$ is the Fermi-Dirac distribution function, and $F_{n m}^{i j}=\psi_{n}^{*}\left(\boldsymbol{r}_{i}\right) \psi_{n}\left(\boldsymbol{r}_{j}\right) \psi_{m}^{*}\left(\boldsymbol{r}_{j}\right) \psi_{m}\left(\boldsymbol{r}_{i}\right)$, with $\psi_{n}\left(\boldsymbol{r}_{i}\right)$ denoting the eigenfunction corresponding to the eigenenergy $E_{n}$ of the electronic disorder-free Hamiltonian. The lattice constant $a$ and the Planck's constant $\hbar$ are set to unity in all numerical calculations. Using a zero-temperature approximation $(T=0)$ and changing to an integral form, Eq. (2) can be recast as

$$
J_{\mathrm{RKKY}}=-J^{2} \frac{S(S+1)}{2 S^{2}} \int_{E<\mu} d E \int_{E^{\prime}>\mu} d E^{\prime} \frac{F\left(E, E^{\prime}\right)}{E-E^{\prime}},
$$


(a)

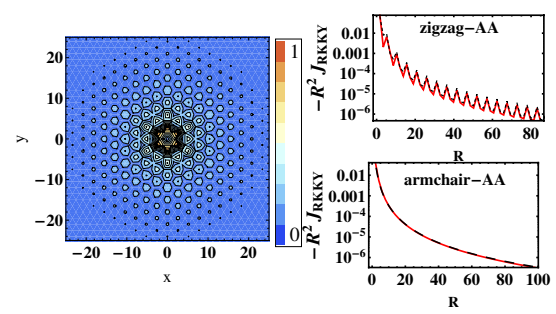

(b)

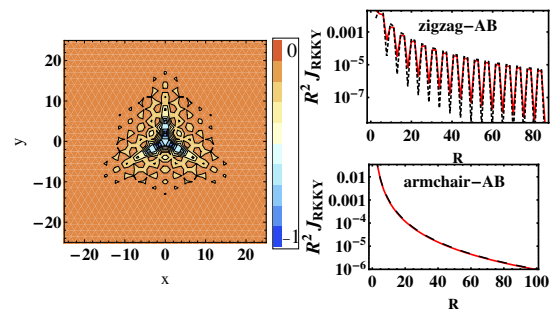

Fig. 2. Plots of the RKKY interaction strengths between a magnetic impurity at the origin and another at: (a) a site from the same sublattice (AA) and (b) a site from a different sublattice (AB). The amplitudes are multiplied by the square of the distance to facilitate visualization. The lattice constant is set to unity. The numerical data is for clean graphene $(W=0)$. Calculations using the kernel polynomial method and lattice Green's function method are represented as solid black and dashed red lines, respectively.

where $F\left(E, E^{\prime}\right)=\operatorname{Re}\left[\rho_{j i}(E) \rho_{i j}\left(E^{\prime}\right)\right], \mu$ is the Fermi energy, and $\rho_{i j}(E)=\langle i| \delta(E-$ $H)|j\rangle$, which can be calculated numerically using the kernel polynomial method (KPM). ${ }^{6,7}$ In the KPM, the matrix elements $\rho_{i j}(E)$ are expressed as sums over order- $M$ Chebyshev polynomials on the energy $E$ with coefficients obtained through an efficient recursion relation involving matrix elements of the system Hamiltonian. As our unperturbed electronic Hamiltonian with on-site disorder, we employ the single-band Anderson tight-binding model on a honeycomb lattice,

$$
H=-t \sum_{\langle i, j\rangle} c_{i}^{+} c_{j}+\sum_{i} w_{i} c_{i}^{+} c_{i}
$$

where $t\left(\approx 2.67 \mathrm{eV}\right.$ for graphene) is the hopping energy, $c_{i}\left(c_{i}^{+}\right)$annihilates (creates) an electron at site $i, w_{i}$ is the on-site random disorder energy distributed uniformly between $[-W / 2, W / 2]$, and $\langle i, j\rangle$ denote nearest-neighbor sites. Periodic boundary condition is used for all calculations.

\section{Numerical results}

\subsection{Clean system}

For pure systems $(W=0)$ at the neutrality point $(\mu=0)$, the Chebyshev polynomials are calculated up to $M=3 \times 10^{3}$ on a lattice with $5 \times 10^{5}$ sites and the numerical results are shown in Fig. 2, where the $R^{2}$ are multiplied to emphasis the oscillatory

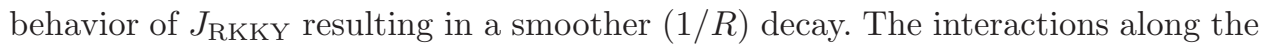
zigzag and armchair directions are shown separately by line plots in Fig. 2. These results are in excellent agreement with previous studies. ${ }^{1-3}$ The authors of Ref. 3 
(a)

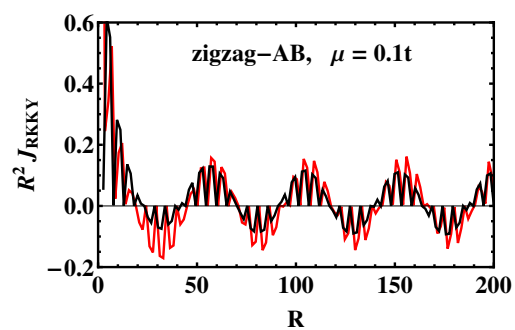

(b)

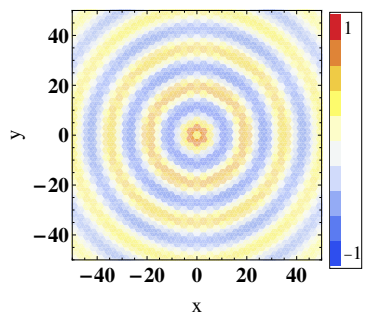

Fig. 3. The RKKY interaction multiplied by $R^{2}$ in doped graphene (a) $\mu=0.1 t$ along zigzag$\mathrm{AB}$, density plots for (b) $\mu=0.3 t$, where $t$ is the nearest-neighbor hopping energy. The lattice constant is set to unity. Calculations using the kernel polynomial method and lattice Green's function method are represented as solid blue and dashed red line respectively in (a). A lattice with $7.2 \times 10^{5}$ and a polynomial degree cut off of $M=5 \times 10^{3}$ are used in these numerical calculations. The lattice constant $a$ is set to unity.

used a lattice Green's function method to obtain an RKKY interaction of the form

$$
\begin{aligned}
& J_{\mathrm{AA}}^{0}=-J^{2} \frac{1+\cos \left[\left(\boldsymbol{K}-\boldsymbol{K}^{\prime}\right) \cdot \boldsymbol{R}\right]}{R^{3}} \\
& J_{\mathrm{AB}}^{0}=J^{2} \frac{3+3 \cos \left[\left(\boldsymbol{K}-\boldsymbol{K}^{\prime}\right) \cdot \boldsymbol{R}+\pi-2 \theta_{R}\right]}{R^{3}},
\end{aligned}
$$

where all the coefficients are set to unity, $\mathbf{R}=\mathbf{r}_{i}-\mathbf{r}_{j}$, and $\theta_{R}$ is defined in Fig. 1a. For a direct comparison, plots of Eqs. (5) and (6) are shown in Fig. 2 along with the results calculated from Eq. (3). As expected from the particle-hole symmetry of the spectrum, the magnetic impurity on the origin has ferromagnetic correlations with the impurities on the same sublattice (Fig. 2a), while antiferromagnetic correlations develop for impurities on different sublattices (Fig. 2b).

By controlling $\mu$ in Eq. (3), we investigate how the RKKY interaction evolves with the Fermi level and the results are shown in Fig. 3, where the $R^{2}$ are multiplied to emphasize the oscillatory behavior of $J_{\mathrm{RKKY}}$. We have used a lattice with $7.2 \times 10^{5}$ and a polynomial degree cut off of $M=3 \times 10^{3}$ in these KPM calculations. Near the Dirac point (Fig. 3a, b), beating pattern appears and it consists of the waves which are characterized by two wave vectors, $\boldsymbol{K}-\boldsymbol{K}^{\prime}$ and $\boldsymbol{q}_{F}$, where $\boldsymbol{q}_{F}$ is the Fermi wave vector. With electron or hole doping the particle-hole symmetry is broken, so that it causes the sign alteration with the distance between the moments. Recently, for this beating behavior, analytical expression using the lattice Green's function has been reported, ${ }^{4}$

$$
\begin{aligned}
& J_{\mathrm{AA}}=J_{\mathrm{AA}}^{0}\left[1+\frac{8 q_{F} R}{\sqrt{\pi}} G_{1,3}^{2,0}\left(\begin{array}{c}
\frac{1}{2}, \frac{3}{2} \\
1,1,1
\end{array} ; q_{F}^{2} R^{2}\right)\right], \\
& J_{\mathrm{AB}}=J_{\mathrm{AB}}^{0}\left[1-\frac{8 q_{F} R}{\sqrt{3 \pi}} G_{2,4}^{2,1}\left(\begin{array}{c}
\frac{1}{2}, \frac{3}{2} \\
1,2,0,-\frac{1}{2}
\end{array} ; q_{F}^{2} R^{2}\right)\right] \text {, }
\end{aligned}
$$


(a)

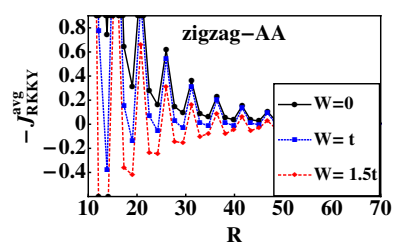

(b)

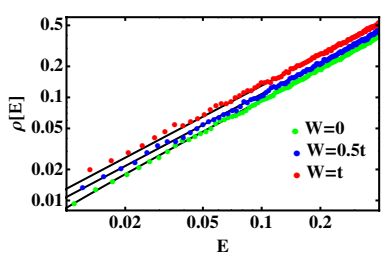

(c)

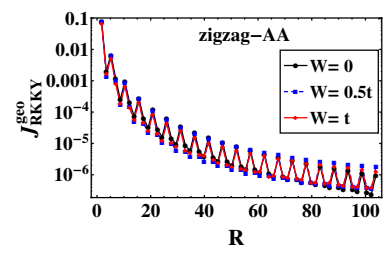

Fig. 4. Plots of the RKKY interaction strength along the zigzag in the diffusive regime, as (a) averaged and (b) geometrical averaged over 1600 different disorder configurations. A lattice with $1.8 \times 10^{5}$ sites and a polynomial degree cutoff of $M=5 \times 10^{3}$ are used in these numerical calculations. (c) The averaged density of states in log scales, where a lattice with $3 \times 10^{7}$ sites and a polynomial degree cutoff of $M=7 \times 10^{3}$ are used. The black lines in (c) represent fittings to the relation $\rho(E)=\gamma|E|$ with $\gamma=0.95,1.07,1.3$ for $W=0,0.5 t, t$, respectively

where $J_{\mathrm{AA}(\mathrm{B})}^{0}$ is the coupling function at Dirac point [Eq. (5),(6)], $G$ is the Meijer-G function. Note that the expression describing the oscillatory behavior with respect to the Fermi momentum $\boldsymbol{q}_{F}$, depends only on its magnitude $q_{F}$ not its direction, contrary to the $J_{\mathrm{AA}(\mathrm{B})}^{0}$ which depends on both the direction and magnitude of the interference of the two neighbor Dirac point $\boldsymbol{K}-\boldsymbol{K}^{\prime}$. To compare with our calculation, the result of Eq. (8) is also presented by black solid line in Fig. 3a and these are in excellent agreement each other.

\subsection{Disordered system}

In order to investigate the effect of on-site nonmagnetic disorder in graphene, we consider 1600 different disorder configurations for each value of $W$ and then evaluate the matrix elements $\rho_{i j}$ through the KPM with $M=5 \times 10^{3}$ on a lattice with $1.8 \times 10^{5}$ sites. For weak disorder strength, the system is in the diffusive regime; the actual value of $W$ where this crossover occurs depends on the lattice size and it has been determined by evaluating the localization length. The average amplitude of the RKKY interaction in the diffusive regime is shown in Fig. 4a. Similarly to conventional metals, the interaction decays exponentially with increasing disorder strength as $J_{\mathrm{RKKY}}^{\mathrm{avg}} \sim J_{\mathrm{RKKY}}^{\text {clean }} e^{-R / l_{e}}$, where $l_{e}$ is the mean free path and $J_{\mathrm{RKKY}}^{\text {clean }}$ is the interaction amplitude in the clean limit. It is worth noticing that the sign of the interaction oscillates when the impurities are located along the zigzag AA direction.

To better characterize the amplitude of the interaction, we have also calculated the geometrical average $\left(J_{\mathrm{RKKY}}^{\text {geo }}\right)$ for diffusive regimes (Fig. $\left.4 \mathrm{~b}\right)$. We observed that the geometrical average for weakly disordered system has a decaying behavior similar to the clean system, as previous works expected. ${ }^{8}$ We have calculated the density of state for two disordered system $(W=0.5 t, t)$ to observe how the weak disorder affects the pseudo gap at the Dirac point $(E=0)$ which leads to the unconventional decaying behavior of the RKKY interaction $\left(1 / R^{3}\right)$. For the density of state calculation, the KPM has been also used with a $3 \times 10^{7}$ sites and a polynomial degree cutoff 
of $M=7 \times 10^{3}$. As one may see from the density of state calculation (Fig. 4c), the pseudo gap, $\rho(E)=\gamma|E|$, is still not filled with weak disorder and only the slope $(\gamma)$ around the Dirac point is changed, which means that the geometrical average of the RKKY interaction may not change drastically from the clean limit.

\section{Conclusion}

In conclusion, we have confirmed that the RKKY interactions in clean graphene has a strong anisotropy of its sign and oscillation amplitude, and it decays as $1 / R^{3}$ for all directions. We also have studied the effect of the gate voltage and non magnetic disorder on the RKKY interaction with the KPM. The finite gate voltage breaks the particle-hole symmetry and gives the finite Fermi surface which yields the Friedel oscillation behavior in long rage limit, so that the sign of the RKKY interaction between the impurities localized on the same sublattice oscillates with distance. Increasing the strength of nonmagnetic, on-site disorder causes the averaged amplitude of the RKKY interaction to decrease exponentially at distances exceeding the elastic mean free path. With the accurate evaluation of the density of states around the neutrality point in weakly disordered regime $(W \leq t)$, we have confirmed that the linear relation is still valid and the pseudo gap is not filled. Therefore, we may conclude that the geometrical average of the RKKY interaction in diffusive regime decays with $1 / R^{3}$ as in the clean system, not $1 / R^{2}$ as in the usual 2 dimensional metal.

\section{Acknowledgments}

This research was supported by WCU(World Class University) program through the National Research Foundation of Korea funded by the Ministry of Education, Science and Technology(R31-2008-000-10059-0)

\section{References}

1. S. Saremi, Phys. Rev. B 76, 184430 (2007).

2. A. M. Black-Schaffer, Phys. Rev. B 81, 205416 (2010).

3. M. Sherafati and S. Satpathy, Phys. Rev. B 83, 165425 (2011).

4. M. Sherafati and S. Satpathy, Phys. Rev. B 84, 125416 (2011).

5. R. M. White, Quantum Theory of Magnetism (McGraw-Hill, New York, 1970).

6. Roche, Stephan and Mayou, Didier, Phys. Rev. B. 60, 322 (1999).

7. A. Weiße, G. Wellein, A. Alvermann, and G. Fehske, Rev. Mod. Phys. 78, 275 (2006).

8. P. F. de Chatel, J. Magn. Magn. Mater. 23, 28 (1981).

9. H. Lee, E. R. Mucciolo, G. Bouzerar and S. Kettemann, Phys. Rev. B 85, 075420 (2012)

10. H. Lee, E. R. Mucciolo, G. Bouzerar and S. Kettemann, unpublished. 